This is a post-peer-review, pre-copyedit version of an article published in Proceedings of the IEEE Conference on Nanotechnology. The final authenticated version is available online at: https://dx.doi.org/10.1109/NANO.2018.8626244. (c) 2019 IEEE. Personal use of this material is permitted.

Permission from IEEE must be obtained for all other uses, in any current or future media, including reprinting/republishing this material for advertising or promotional purposes, creating new collective works, for resale or redistribution to servers or lists, or reuse of any copyrighted component of this work in other works. 


\title{
On the enhancement of the thermal conductivity of graphene-based nanofluids
}

\author{
M. R. Rodriguez-Laguna ${ }^{1,2}$, C.M. Sotomayor Torres ${ }^{1,3}$, P. Gomez-Romero', E. Chavez-Angel*1 \\ ${ }^{1}$ Catalan Institute of Nanoscience and Nanotechnology (ICN2), CSIC and BIST, Campus UAB, Bellaterra, 08193 Barcelona, Spain e- \\ mail: emigdio.chavez@icn2.cat \\ ${ }^{2}$ Dept. Chemistry, Universitat Àutonoma de Barcelona, Campus UAB, Bellaterra, 08193 Barcelona, Spain \\ ${ }^{3}$ ICREA, Pg. Lluís Companys 23, 08010 Barcelona, Spain
}

\begin{abstract}
Heat transfer fluids have been extensively used in both low-temperature and high temperature applications (e.g. microelectronics cooling and concentrated solar power). However, their low thermal conductivity is still a limit on performance. One way to enhance thermal properties is to disperse nanomaterials, such as graphene flakes in the base fluid. In this work, we have developed highly stable DMAc-graphene nanofluids with enhanced thermal properties. Furthermore, the displacement of several Raman bands as a function of graphene concentration in DMAc suggests that the solvent molecules are able to interact with graphene surfaces strongly
\end{abstract}

\section{INTRODUCTION}

Recent advances in electronic technology have been directed toward miniaturizing components and achieving faster performances, causing an increment of power densities. Consequently, the thermal energy increases and temperature control becomes a crucial challenge. If the device is not cooled down, the reliability and performance of electronic components will be negatively affected[1]. Various cooling systems have been used in electronics, such as forced air cooling, direct liquid immersion, forced convection liquid-cooling, and those based on a phase change of the coolant e.g. heat pipes and phase change materials[1][3]. A major problem of conventional coolants is the low heat exchange rate and the thermal conductivity, which is too low to meet the coming needs and challenges in the field. One way to overcome this barrier is by using solid nanoparticles dispersed in fluids, i.e. nanofluid (NF). The presence of well dispersed nanoparticles in a fluid can provide remarkable improvements in thermal properties, such as thermal conductivity[4]-[6] and in some cases, specific heat capacity[7].

In this work graphene-based NFs were prepared using dimethylacetamide (DMAc) as a solvent. This organic compound is able to disperse graphene easily, forming stable dispersions over time without the need of using any surfactant to stabilize the flakes[8]. In addition, this solvent is liquid in a wider temperature range $\left(-20^{\circ} \mathrm{C}\right.$ to $\left.165^{\circ} \mathrm{C}\right)$. This fact permits the use of these fluids in a wider working temperature range than most used coolant: water.

\section{MethodologY}

\section{A. Sample preparation}

Graphene flakes with sizes $~ 150-450 \mathrm{~nm}$ were prepared from graphite (Sigma-Aldrich, purity $>99+\%$ and size $<20$ um) by mechanical exfoliation using a novel method developed in our laboratories [9]. The stability was monitored by using dynamic light scattering (DLS). The samples were analyzed periodically to register the number of photons able to cross the sample (one measurement per month for four months).

\section{B. Thermal conductivity of Nanofluids}

The thermal conductivity $(k)$ measurements were measured by using a modified version of the common three-omega $(3 \omega)$ method suitable for the measurement of liquids[10], [11]. As is shown in Fig. 1, the liquid is placed on top of the $3 \omega$-heater which has been passivated with $200 \mathrm{~nm}$ of $\mathrm{SiO}_{\mathrm{x}}$ to avoid current

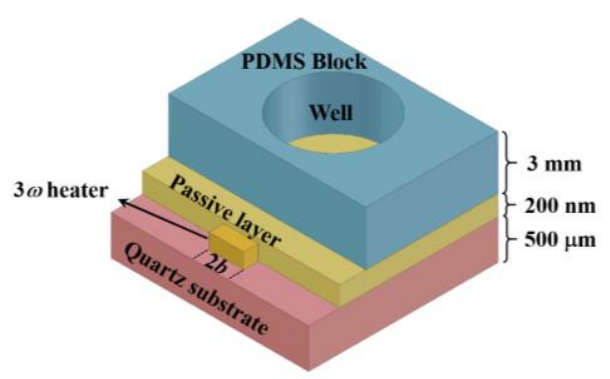

Fig. 1. Schematic representation of three-omega cell (no to scale). leakage from the resistor to the conductive fluid. A $100 \mathrm{~nm}$ thick chromium-gold $3 \omega$-strip was patterned on a $0.5 \mathrm{~mm}$ thick quartz substrate by photolithography and electron beam physical vapor deposition (EBPVD). The width of the heating line is defined as $2 b=10 \mu \mathrm{m}$ and the length as $l=1 \mathrm{~mm}$, the latter considered as the distance between the voltage (inner) pads. Then, $3 \mathrm{~mm}$ thick PDMS block is used to seal the circuit and as liquid container (well). 


\section{Raman mesurements}

The Raman spectra were recorded by T64000 Raman spectrometer manufactured by HORIBA Jobin Yvon. It was used in single grating mode with a spectral resolution better than $0.4 \mathrm{~cm}^{-1}$. All the measurements were carried out by focusing a diode laser $(532 \mathrm{~nm})$ with 50x long working distance microscope objective and using a transparent quartz cuvette. The power of the laser was kept as low as possible $(\sim 1 \mathrm{~mW})$ to avoid any possible effect from self-heating.

\section{RESUltS}

Fig. 2 shows the temperature dependence of $k$ for pure DMAc and two different concentrations of graphene NFs (G-DMAc). The thermal conductivity of the NFs shows an enhancement of $k$ as the graphene concentration increases. While the enhancement of $k$ has been commonly observed in other NFs[4]-[6]. The mechanism behind of this phenomenon has not yet been elucidated. However, our Raman results show that the enhancement of thermal energy transfer can be associated to increased interatomic interactions.

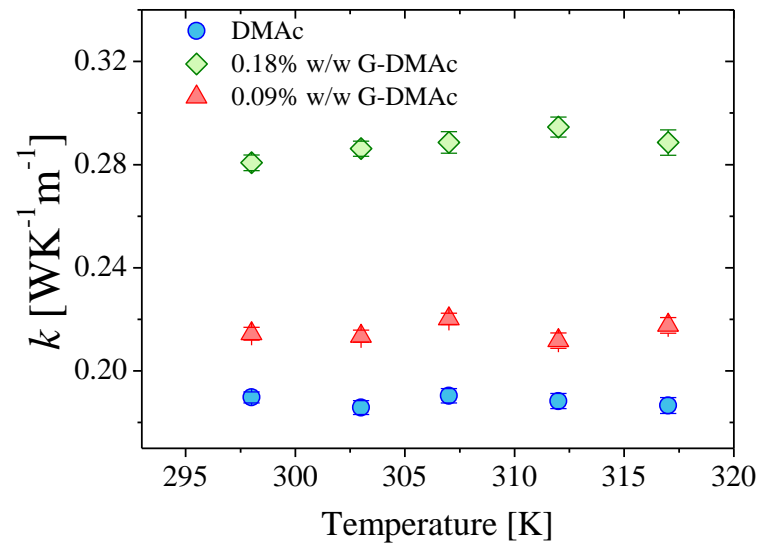

Fig. 2. Comparison of thermal conductivity as a function of temperature for: DMAc (blue circles), $0.09 \%$ (red triangles) and

$0.18 \% \mathrm{w} / \mathrm{w}$ (green squares) graphene-DMAc nanofluids.
As it is shown in Fig. 3, the displacement of two Raman bands as a function of graphene concentration suggests that the solvent molecules are able to interact with graphene surfaces strongly, affecting the overall Raman frequencies. The blue shift of several Raman bands with increasing graphene concentration in DMAc suggests an overall modification of the intermolecular interactions in the solvent. These observations are supported density functional theory and molecular dynamics simulations, indicating that there is a strong interaction between graphene flakes and the nearest DMAc molecules around them, suggesting a possible $\pi-\pi$ stacking.

\section{ACKNOWLEDGMENT}

We acknowledge support from the Severo Ochoa Program (MINECO, Grant SEV-2013-0295) and funding from the CERCA Programme/Generalitat de Catalunya. Funding from the Spanish Ministry (MINECO-FEDER MAT2015-68394-R NaCarFLOW) and PHENTOM (FIS2015-70862-P) are acknowledged.

\section{REFERENCES}

[1] L. C. Lv and J. Li, "Micro Flat Heat Pipes for Microelectronics Cooling: Review," Recent Patents Mech. Eng., vol. 999, no. 999, pp. 1-6, Sep. 2013.

[2] S. M. Sohel Murshed and C. A. Nieto de Castro, "A critical review of traditional and emerging techniques and fluids for electronics cooling," Renew. Sustain. Energy Rev., vol. 78, pp. 821-833, Oct. 2017.

[3] L. M. Poplaski, S. P. Benn, and A. Faghri, "Thermal performance of heat pipes using nanofluids,” Int. J. Heat Mass Transf., vol. 107, pp. 358-371, Apr. 2017.

[4] S. U. S. Choi and J. A. Eastman, "Enhancing thermal conductivity of fluids with nanoparticles," ASME Int. Mech. Eng. Congr. Expo., vol. 66, no. March, pp. 99-105, 1995

[5] M. Patil, S. Kim, J. Seo, and M. Lee, "Review of the Thermo-Physical Properties and Performance Characteristics of a Refrigeration System Using

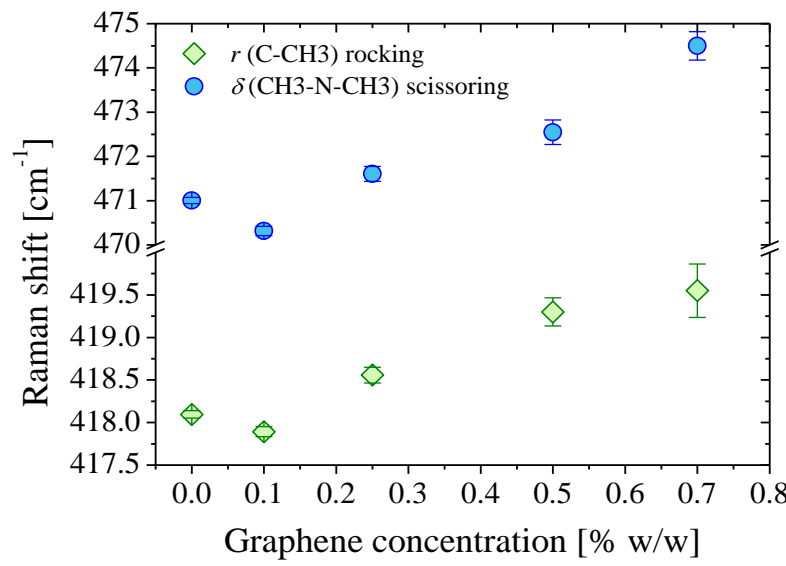

Fig. 3. Peak position of two modes of DMAc for different graphene concentrations. The peak positions were obtained from a Lorentzian fit of the Raman signal. Refrigerant-Based Nanofluids," Energies, vol. 9, no. 1, p. 22, Dec. 2015.

[6] W. H. Azmi, K. V. Sharma, R. Mamat, G. Najafi, and M. S. Mohamad, "The enhancement of effective thermal conductivity and effective dynamic viscosity of nanofluids - A review," Renew. Sustain. Energy Rev., vol. 53, pp. 1046-1058, Jan. 2016.

[7] P. Andreu-Cabedo, R. Mondragon, L. Hernandez, R. MartinezCuenca, L. Cabedo, and J. E. Julia, "Increment of specific heat of Solar Salt with $\mathrm{SiO} 2$ and $\mathrm{A} 12 \mathrm{O} 3$ nanoparticles," Nanoscale Res. Lett., vol. 9, no. 1, p. 582, 2014.

[8] C. Shih, S. Lin, M. S. Strano, and D. Blankschtein, "Understanding the Stabilization of Liquid-Phase-Exfoliated Graphene in Polar Solvents: Molecular Dynamics Simulations and Kinetic Theory of Colloid Aggregation," no. 13 , pp. $14638-14648,2010$.

[9] D. P. Dubal and P. Gomez-Romero, "Electroactive graphene nanofluids for fast energy storage," 2D Mater., vol. 3, no. 3, p. 31004, Jul. 2016. [10] F. Chen, J. Shulman, Y. Xue, C. W. Chu, and G. S. Nolas, "Thermal conductivity measurement under hydrostatic pressure using the $3 \omega$ method," Rev. Sci. Instrum., vol. 75, no. 11, pp. 4578-4584, Nov. 2004.

[11] D. W. Oh, A. Jain, J. K. Eaton, K. E. Goodson, and J. S. Lee, "Thermal conductivity measurement and sedimentation detection of aluminum oxide nanofluids by using the $3 \omega$ method," Int. J. Heat Fluid Flow, vol. 29, pp. 1456 1461,2008 
